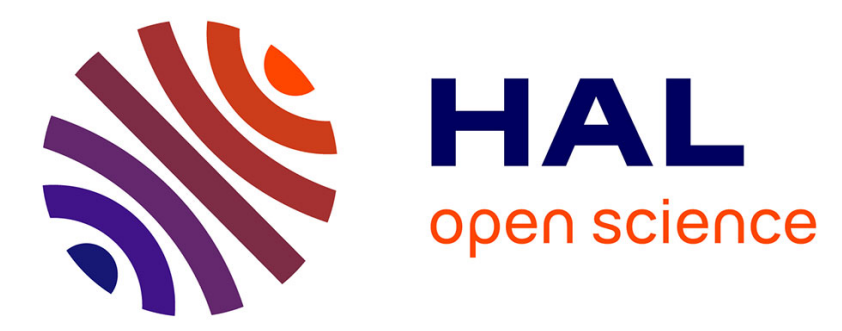

\title{
Time-gated triplet-state optical spectroscopy to decipher organic luminophores embedded in rigid matrices
}

\author{
Atul D. Sontakke, Jean-Marie Mouesca, Victor Castaing, Alban Ferrier, \\ Mathieu Salaün, Isabelle Gautier-Luneau, Vincent Maurel, Alain Ibanez, \\ Bruno Viana
}

\section{To cite this version:}

Atul D. Sontakke, Jean-Marie Mouesca, Victor Castaing, Alban Ferrier, Mathieu Salaün, et al.. Timegated triplet-state optical spectroscopy to decipher organic luminophores embedded in rigid matrices. Physical Chemistry Chemical Physics, 2018, 20 (36), pp.23294-23300. 10.1039/C8CP03952F . hal01958712

\section{HAL Id: hal-01958712 \\ https://hal.sorbonne-universite.fr/hal-01958712}

Submitted on 18 Dec 2018

HAL is a multi-disciplinary open access archive for the deposit and dissemination of scientific research documents, whether they are published or not. The documents may come from teaching and research institutions in France or abroad, or from public or private research centers.
L'archive ouverte pluridisciplinaire HAL, est destinée au dépôt et à la diffusion de documents scientifiques de niveau recherche, publiés ou non, émanant des établissements d'enseignement et de recherche français ou étrangers, des laboratoires publics ou privés. 


\title{
Time-gated triplet-state optical spectroscopy to decipher organic luminophores embedded in rigid matrices $\dagger$
}

\author{
Atul D. Sontakke, (D)*a Jean-Marie Mouesca, (D) ${ }^{b}$ Victor Castaing, ${ }^{a}$ Alban Ferrier, ${ }^{a c}$ \\ Mathieu Salaün, ${ }^{d}$ Isabelle Gautier-Luneau, (D) ${ }^{d}$ Vincent Maurel, (iD b ${ }^{b}$ Alain Ibanez ${ }^{d}$ \\ and Bruno Viana (D) *a
}

\begin{abstract}
Wet-chemically synthesized inorganic materials often exhibit luminescence behavior. We have recently shown that the amorphous yttrium-aluminium-borate (a-YAB) powders obtained by sol-gel and modified Pechini methods exhibit organic impurities, responsible for their intense visible photoluminescence and phosphorescence afterglow. However, the heterogeneity of impurity organic compounds and difficulties in their intact extraction from the solid inorganic host matrix limit the extraction-based chemical analysis for luminophore identification. Here, we propose a photo-physical route based on time-gated tripletstate optical spectroscopy (TGTSS) to construct the electronic structures of the trapped unknown luminophores, which successfully illustrates the luminescence properties of a-YAB powders in more detail and also provides important insights intrinsic to the nature of the luminophores. The experimental results accompanied with TD-DFT calculations of the theoretical electronic structures thus help us to propose the probable luminophore compounds trapped in rigid a-YAB matrices. We anticipate that the present approach will open new opportunities for analyzing similar complex luminescent materials, including carbon dots, graphene oxides, etc., which is vital for their improvement.
\end{abstract}

\section{Introduction}

Organic compounds in solution and vapor phases are rather easy to distinguish using state-of-the-art analytical techniques even in trace amounts. In rigid matrices, however, the molecular heterogeneity added with its complex bonding within rigid organic-inorganic frameworks unfavorably reduces the detection sensitivity, which then depends on their extraction yield from the host matrix. ${ }^{1,2}$ Chemical extraction is highly controlled by the matrix properties, such as its ability to get dissolved in organic solvents or corrosive reactants (mild acids, bases, etc.). In the case of inorganic matrices, the requirements of relatively harsh reactants complicate the extraction of organic compounds in their original form. ${ }^{3}$ This reduces the reliability of extraction based analytical techniques, thereby restricting

\footnotetext{
${ }^{a}$ PSL Research University, Chimie ParisTech - CNRS, Institut de Recherche de Chimie Paris, 75005 Paris, France.E-mail: atul.sontakke@chimie-paristech.fr, bruno.viana@chimie-paristech.fr

${ }^{b}$ Univ. Grenoble Alpes, CEA, CNRS, INAC, SyMMES, F-38000 Grenoble, France

${ }^{c}$ Sorbonne Université, Faculté des Sciences et Ingénierie, UFR 933, F-75005 Paris, France

${ }^{d}$ Univ. Grenoble Alpes, CNRS, Grenoble INP, Institut Néel, 38000 Grenoble, France $\dagger$ Electronic supplementary information (ESI) available. See DOI: 10.1039/ c8cp03952f
}

their analysis to less precise solid-state methods. The analyses become even more challenging if one has to deal with particular functional molecules amongst heterogeneous molecular crowd strongly confined in rigid host framework.

We experienced similar difficulty in pinpointing the luminescent centers trapped in amorphous Yttrium-AluminiumBorate (a-YAB) matrices. These a-YAB micrometer-sized powders prepared by modified Pechini and sol-gel methods give intense visible emissions with potential for developing metal ion-free luminescent materials, particularly phosphors for solid-state lighting. ${ }^{4-6}$ Such intense visible luminescence has also been reported in many other inorganic materials obtained by various wet-chemical routes. ${ }^{7-11}$ During the synthesis process, the metal-organic precursors undergo a complex chain of reactions, including polyesterification (Pechini route) or polycondensation (sol-gel chemistry) reactions for the preparation of initial resins or gels, followed by degradation and the associated structural transformation during the thermal treatments performed under controlled atmospheric conditions (pyrolysis or calcination). This leads to a variety of organic clusters trapped inside the solid inorganic matrix. Indeed, we showed by differential thermal analysis and thermogravimetry, coupled with mass spectrometry, that the $\mathrm{a}-\mathrm{YAB}$ powders contain significant amounts of carbonaceous (organic) compounds (200-10000 ppm) arising from 
precursors and solvents. ${ }^{4,5}$ Moreover, we were able to make progress in establishing a correlation of photoluminescence (PL) emission with the presence of aromatic compounds such as small polycyclic aromatic hydrocarbon (PAH) molecules based on the observations of solid-state NMR, EPR-ENDOR and associated DFT calculations. ${ }^{4}$ However, still precise information on luminescent compounds is not fully at reach, which is crucial in improving the performance of these promising metal ion-free luminescent materials.

Besides analytical methods, the photo-physical studies can be of great use to resolve the electronic structure and luminescence mechanism of the luminophores. The steady-state PL reveals excitation-emission characteristics, which is informative to understand the preliminary electronic structure of the luminophores. Unfortunately, a vast amount of organic compounds are luminescent in nature and differentiating them based on mere PL-PLE (PL excitation) or decay characteristics is simply impossible. Nevertheless, additional details about the location of singlet $\left(\mathrm{S}_{\mathrm{i}}\right)$ and triplet $\left(\mathrm{T}_{\mathrm{i}}\right)$ states, the localized or delocalized nature of excited states, the singlet-triplet energy difference $\left(\Delta E_{\mathrm{ST}}\right)$, excited state dynamics, etc., could certainly narrow down the search and enrich the understanding of the trapped luminescent centers. This additional information provides better prospects for experimental and/or theoretical studies tallying with known probable luminophore candidates. Time-gated spectroscopy reveals the triplet-state dynamics, ${ }^{12}$ which coupled with steady-state spectroscopy, can allow constructing detailed electronic structure and obtaining important insights on the excited state dynamics of the luminophores. Moreover, time-gated spectroscopy unveils weak effects, which are otherwise masked due to strong fluorescence in steady-state measurements. The triplet-state efficiency is generally weak at ambient temperature, but in rigid matrices, it is rather stabilized and yields efficient phosphorescence even at room temperature. ${ }^{13}$ In a-YAB powders, the afterglow emission lasts for several seconds at room temperature (RT). ${ }^{6}$

\section{Results and discussion}

Fig. 1a shows the steady-state PL-PLE contour plot of SG-450 a-YAB powder, synthesized from a sol-gel resin calcined at $450{ }^{\circ} \mathrm{C}$. The powder exhibits blue PL emission corresponding to

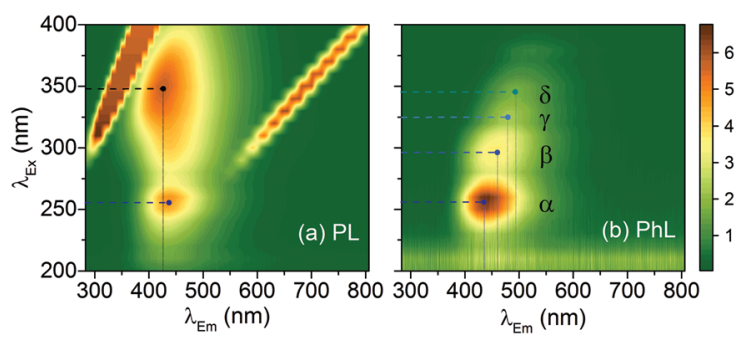

Fig. 1 Steady-state (a) and time-gated (b) luminescence contour maps of SG-450 a-YAB powder. In (a), the diagonal features are excitation pump signals. In the time-gated plot (b), data represent the emission signals at $1 \mathrm{~s}$ after ceasing the excitation. The intensity scale bar is presented on the right side. two distinct excitation contours peaking at about $255 \mathrm{~nm}$ and $350 \mathrm{~nm}$. A careful observation reveals that the $350 \mathrm{~nm}$ PLE contour is non-symmetric and the peak maximum points to a relatively shorter PL wavelength than for the $255 \mathrm{~nm}$ PLE contour. This represents an important revelation about the emitting centers and their mechanism, however it is not possible to resolve it more from the steady-state luminescence properties. To gain better insights, the time-gated afterglow emission (PhL) was recorded and presented as the PhL-PhLE (PhL excitation) contour plot in Fig. 1b. Unlike the steady-state, the afterglow emission contour exhibits intense $255 \mathrm{~nm}$ excitation monitoring the blue emission but the corresponding $350 \mathrm{~nm}$ excitation is missing. This suggests that only high-energy excitation is contributing to the afterglow emission. Moreover, additional excitation contours are present corresponding to afterglow emissions at longer wavelengths with decreasing strengths. These weak excitation contours peaking at about $295 \mathrm{~nm}, 325 \mathrm{~nm}, 345 \mathrm{~nm}$, etc., suggest that the $\mathrm{a}-\mathrm{YAB}$ powder exhibits multiple luminescent centers coexisting at different proportions and are denoted as $\alpha, \beta, \gamma$, $\delta$, etc., as in Fig. $1 \mathrm{~b}$.

Among the different luminescent centers, the $\alpha$ ones are most dominant in SG-450 powder, followed by $\beta, \gamma, \delta$, etc., with decreasing weights. Since the additional luminophores' transitions overlap the lower energy excitation of $\alpha$ centers, the PLE contour exhibits a non-symmetrical nature in this region (Fig. 1a). Moreover, this also shifts the contour peak position, which therefore represents the convoluted peak due to multiple contributions. Monitoring the PL higher energy edge region at 380-400 nm in Fig. 1a, which is less influenced by additional longer wavelength emitting luminophores, reveals the actual PLE positions of $\alpha$ emitting centers. Accordingly, the $\alpha$ centers exhibit excitations at $255 \mathrm{~nm}\left(\mathrm{~S}_{2}\right)$ and $330 \mathrm{~nm}\left(\mathrm{~S}_{1}\right)$.

Another interesting observation that can be made from Fig. 1 is that the steady-state PL and afterglow emission of SG-450 powder under $255 \mathrm{~nm}$ excitation exhibit similar profiles but differ when excited at lower energy excitations (Fig. 2a). ${ }^{6}$ It is critical to correctly analyze the PL and afterglow emission in luminescent centers, which otherwise mislead the origin of the afterglow mechanism. In organic luminophores, the slow afterglow can be caused by the spin-forbidden triplet-to-singlet $\left(\mathrm{T}_{1} \rightarrow \mathrm{S}_{0}\right)$ transition, thermally-assisted delayed fluorescence (TADF) or inverted singlet-triplet (iST), also known as triplettriplet annihilation mechanisms (ESI, $\dagger$ Scheme S1). ${ }^{13-15}$ Among them, the first mechanism represents a pure phosphorescence transition from the triplet excited state to the singlet ground state, whereas the latter two mechanisms are effectively singletsinglet $\left(\mathrm{S}_{1} \rightarrow \mathrm{S}_{0}\right)$ transitions delayed by reverse intersystem crossing (rISC). This incorporates a fundamental difference to distinguish these processes experimentally. The phosphorescence $\left(\mathrm{T}_{1} \rightarrow \mathrm{S}_{0}\right)$ transition generally occurs at a longer wavelength with respect to the fluorescence $\left(\mathrm{S}_{1} \rightarrow \mathrm{S}_{0}\right)$ transition following the classical Hund's rule, but the TADF and iST mechanisms exhibit the same fluorescence and delayed afterglow emission profiles owing to their identical origin $\left(\mathrm{S}_{1} \rightarrow \mathrm{S}_{0}\right) \cdot{ }^{16}$ Interestingly, in SG-450, the PL and afterglow emission profiles look similar under $255 \mathrm{~nm}$ excitation (Fig. 2a), thus hinting the presence of 

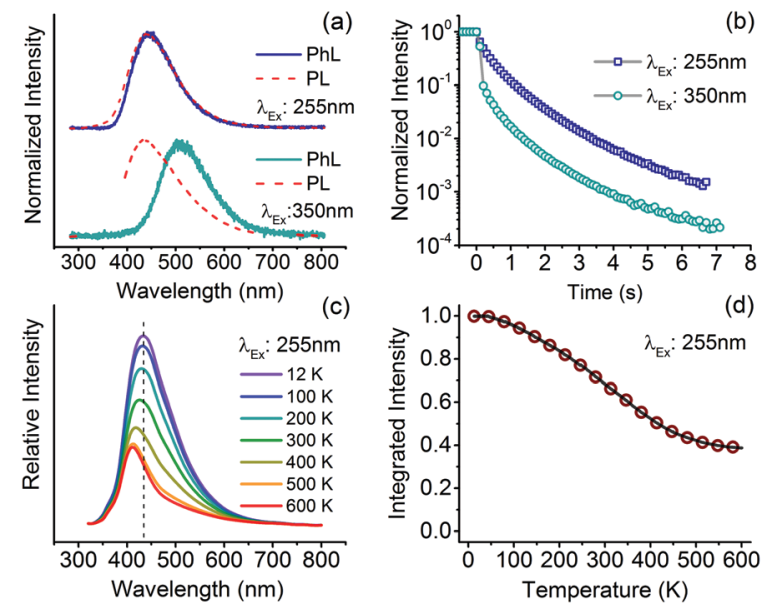

Fig. 2 Steady-state (PL) and time-gated afterglow (PhL) emission spectral profiles of SG-450 powder under $255 \mathrm{~nm}$ and $350 \mathrm{~nm}$ excitations (a); the corresponding afterglow decay kinetics normalized with respect to the steady-state PL (b); temperature dependence of the steady-state PL spectra (c); and the variation of the integrated PL intensity as a function of temperature $(d)$.

the TADF or iST process. However, it is to be noted that the steady-state PL is composed of both prompt fluorescence and slow afterglow emission. The latter is significantly high at RT under $255 \mathrm{~nm}$ excitation, while it is rather weak under $350 \mathrm{~nm}$ excitation (Fig. 2b).

To reveal the peak position of the fluorescence transition, it is necessary to get rid of the afterglow emission in PL signals. This can be achieved in the PL spectra recorded at a higher temperature, as it assists in thermally accelerated diffusion from the excited triplet state to quenching sites. ${ }^{17}$ Fig. 2c shows the temperature-dependent PL spectra of SG-450 powder. At $12 \mathrm{~K}$, the PL peaks at $435 \mathrm{~nm}$ but gradually shifts towards a shorter wavelength with the increase in temperature. This is due to the fact that the PL at low temperature is dominated with slow afterglow emission, but its contribution rapidly vanishes with the increase of temperature, thereby the fluorescence peak resolves more prominently. This is also evident from the PL thermal stability plot (Fig. 2d), exhibiting step-like thermal quenching behavior. The initial continuous decrease in PL up to $400 \mathrm{~K}$ is due to the thermal quenching of afterglow emission contribution. ${ }^{16}$ Above $400 \mathrm{~K}$, the PL attains a relatively stable plateau representing the thermal stability of fluorescence emission. At $600 \mathrm{~K}$, the PL exhibits a prominent peak at $410 \mathrm{~nm}$, suggesting the peak wavelength of the fluorescence transition of $\alpha$ luminescent centers. Considering the thermally influenced red shift, the fluorescence peak at $300 \mathrm{~K}$ may be located at a slightly lower wavelength. This confirms that the slow afterglow is significantly red shifted with respect to fluorescence and is due to the phosphorescence mechanism. The results suggest that the fluorescence $(\sim 405 \mathrm{~nm})$ and phosphorescence $(\sim 440 \mathrm{~nm})$ transitions in the $\alpha$ centers are separated by about $0.25 \mathrm{eV}$, which may account for the singlet-triplet energy difference $\left(\Delta E_{\mathrm{ST}}\right)$. Ideally, $\Delta E_{\mathrm{ST}}$ represents the $\mathrm{S}_{1}-\mathrm{T}_{1}$ energy splitting observed through the zero phonon line (0-0) transitions from the singlet $\left(S_{1} \rightarrow S_{0}\right)$ and triplet $\left(\mathrm{T}_{1} \rightarrow \mathrm{S}_{0}\right)$ states. However, this is rather difficult to measure accurately due to their different degrees of symmetry forbiddenness. ${ }^{18}$ Moreover, in the amorphous solid host, the random variation of dielectric polarization of the surrounding neighborhood leads to a Gaussian-type density of state distribution even at low temperature, unlike in the gas or liquid phase where the vibronic sidebands are vividly distinguishable. ${ }^{17,19}$ Therefore, the peak positions of the fluorescence and phosphorescence transitions are used to estimate the $\Delta E_{\mathrm{ST}}$ value in the present case.

Though the triplet state emission is at a lower energy than the singlet state emission, the triplet state gets populated only by $255 \mathrm{~nm}$ excitation, whereas both $255 \mathrm{~nm}$ and $330 \mathrm{~nm}$ excitation bands contribute to the singlet emission in the $\alpha$ centers. This hints that the intersystem crossing (ISC) coupling interactions of singlet excitation states with the triplet state may not be the same. Fig. 3 shows the diffuse reflectance spectrum of SG-450 powder along with its steady-state PL excitation (PLE) and phosphorescence excitation (PhLE) monitoring the emission peak. The diffuse reflectance spectrum is plotted in the absorption mode for comparison. This shows more resemblance with the phosphorescence excitation spectrum, where the absorption maximum is at about $250 \mathrm{~nm}$ and gradually decreases for longer wavelengths. As the absorption represents the relative strength of transitions, it can be concluded that the oscillator strength of $\mathrm{S}_{2}$ transitions contributing to both PL and phosphorescence is stronger than the $S_{1}$ PLE transition contributing only to the steady-state PL. The high oscillator strength coupled with ease of populating the forbidden triplet state indicates that the $\mathrm{S}_{2}$ state may be delocalized in nature and exhibits a lower binding energy for excited electrons to allow spin flips, thereby favoring an efficient intersystem crossing (ISC) probability to the triplet state. Such properties are intrinsic to charge transfer (CT) or excitonic transitions. ${ }^{13-16,20}$ This suggests that the $255 \mathrm{~nm}$ $\left(\mathrm{S}_{2}\right)$ excitation of the $\alpha$ centers is of delocalized CT nature.

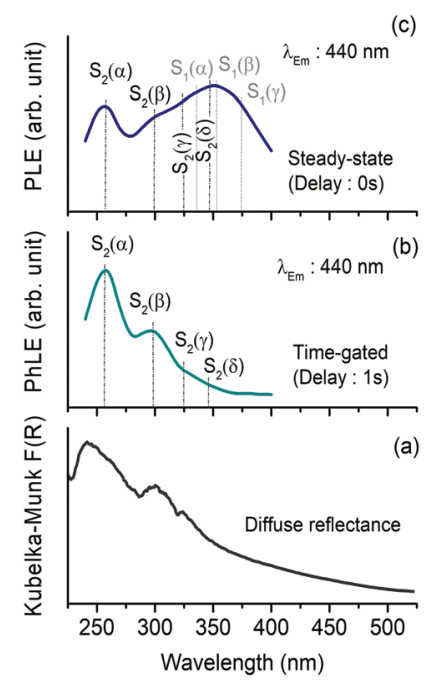

Fig. 3 Absorption spectrum of SG-450 powder (a); the excitation spectrum for phosphorescence (PhLE) (b); and steady-state (PLE) emission (c) monitoring $440 \mathrm{~nm}$ emission. 
Similarly, the $295 \mathrm{~nm}, 325 \mathrm{~nm}, 345 \mathrm{~nm}$, etc. excitation bands contributing to phosphorescence in $\beta, \gamma, \delta$, etc., centers are delocalized $\mathrm{S}_{2}$ states, respectively. This further illustrates why the phosphorescence in SG-450 is significantly red shifted with respect to $\mathrm{PL}$ under $350 \mathrm{~nm}$ excitation and possesses a relatively weak afterglow (Fig. 1 and 2a, b). Accordingly under $350 \mathrm{~nm}$ excitation, the steady-state PL originates from dominant $\alpha$ centers accompanied by small contributions from additional overlapping centers, but the phosphorescence mainly arises from the $\delta$ ones (with small fractions from $\gamma$ and $\varepsilon$ ).

The above results are compiled to construct an energy-level diagram representing the electronic structure of $\alpha$ luminescent centers (Fig. 4). The other luminophores $(\beta, \gamma, \delta$, etc.) do possess a similar energy level scheme but with lower energy transitions. It is evident that the $S_{2}$ state, upon excitation, can populate both $\mathrm{S}_{1}$ and $\mathrm{T}_{1}$ states by internal conversion (IC) and ISC mechanisms, giving rise to fluorescence and phosphorescence, respectively. In contrast, the ISC from the $S_{1}$ state to $T_{1}$ is very weak, thereby only contributing to fluorescence. Moreover, the $\mathrm{T}_{1}$ to $\mathrm{S}_{1}$ rISC is also missing, even $\Delta E_{\mathrm{ST}}$ is only about $0.25 \mathrm{eV}$. Normally, $\Delta E_{\mathrm{ST}}<0.3 \mathrm{eV}$ leads to the TADF process, but in the present case, no TADF is observed in the afterglow emission. This may be due to the fact that the TADF process also requires free dynamical movement between donor (D)-acceptor (A) molecular groups, ${ }^{14,21}$ which might be constrained in the a-YAB matrix owing to the rigid inorganic glassy encapsulation. This suggests that the restricted rISC probability accompanied by low nonradiative losses due to the strong confinement of luminescent centers in rigid matrices is primarily responsible to increase the triplet state excitation lifetime that yields ultra-long phosphorescence even at elevated temperatures.

In an earlier report, we identified the presence of certain organic compounds confined in the a-YAB matrix using solidstate NMR and EPR spectroscopies. ${ }^{4}$ The pulsed-ENDOR associated DFT calculations revealed that the small (2-3 rings) PAH molecules exhibit good correlation with the PL properties of a-YAB powders. ${ }^{4}$ The electronic structures and S-S/S-T excitation energies of these small PAH molecules are calculated here using TD-DFT methods (ESI, $\dagger$ Table S1). Among the several selected compounds, the electronic structure of the phenalene molecule possesses a significant resemblance with the allowed transition of $\alpha$ luminophores presented in Fig. 4. However, the first theoretical triplet-state energy of phenalene is lower than the experimental results. It is to be noted that the calculated molecules are just the simplest working models (based on the

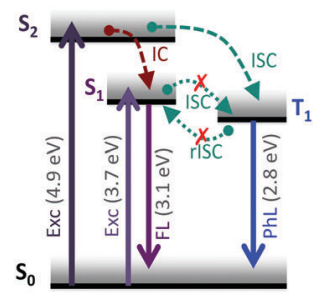

Fig. 4 Jablonski diagram representing the electronic transitions of $\alpha$ centers in SG-450 powder. radical species identified by magnetic resonance techniques), ${ }^{4}$ while the true trapped molecule(s) will most probably be chemically related, exhibiting additional pending functional group(s), which may cause the favorable lift in the triplet-state energy. ${ }^{21}$ The solid host matrix due to its polarization influence also brings a marked reduction in $\Delta E_{\mathrm{ST}}$, associated with the hybrid charge transfer type characters of the singlet-triplet energy levels. ${ }^{22,23}$ The important point here is that phenalene (or chemically-related molecules) satisfies both magnetic (NMR/EPR) resonance measurements and the absorption and PL-PLE experiments.

The evolution of aromatic NMR and ENDOR signals particularly followed the broadening of PL profiles in a-YAB powders synthesized at different temperatures. ${ }^{4}$ Fig. 5a shows the steady-state $\mathrm{PL}$ of a-YAB powders calcined in a temperature range of $450-600{ }^{\circ} \mathrm{C}$. At $450{ }^{\circ} \mathrm{C}$, SG-450 powder exhibits intense blue emission, but the emission becomes broader for higher calcination temperatures and shifts towards the white color with gradual appearance followed by strengthening of longer wavelength emissions. This behavior is related to the evolution and relative strengths of the emitting centers with calcination temperature. In SG-450, the $\alpha$ centers are most prominent leading to intense violet-blue emission. For the powders calcined at higher temperatures, the dominance systematically shifts towards longer wavelength emitting centers, thereby incorporating a gradual change in emission spectrum and PL color coordinates (Fig. 5b). This becomes clear from the PL-PLE contour plots in Fig. 5c and d. The SG-470 sample exhibits distinct contours similar to SG-450 (Fig. 1a), but with more asymmetry in the lower energy region contour. This is due to the fact that the $\beta$ and other additional centers $(\gamma, \delta, \varepsilon$, etc. $)$ are relatively more prominent in SG-470 over SG-450 powder, although the $\alpha$ luminophores are still the most dominant ones. This systematic admixture of
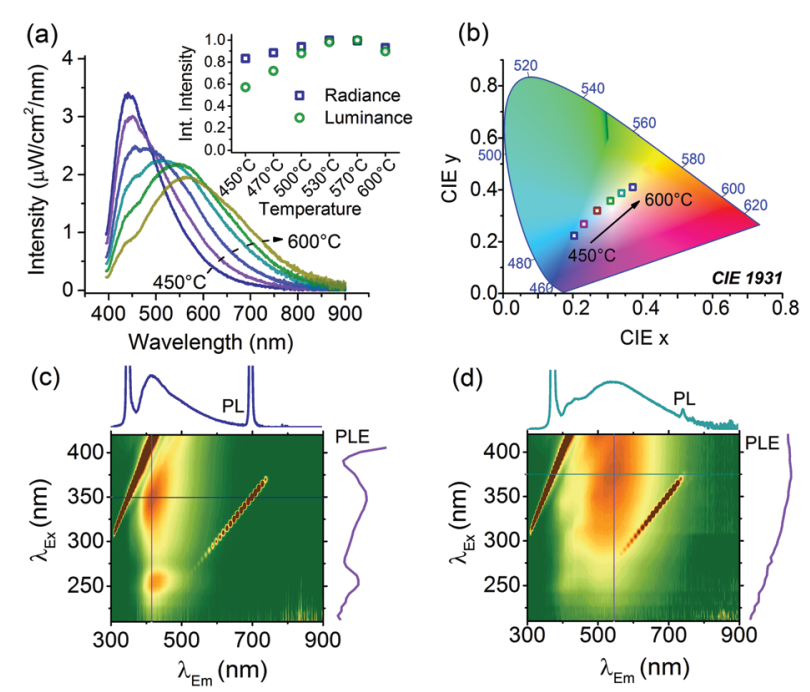

Fig. 5 Steady-state PL spectra of a-YAB powders calcined in a temperature range of $450-600{ }^{\circ} \mathrm{C}$ recorded under $365 \mathrm{~nm}$ excitation (a). The inset indicates the integrated $\mathrm{PL}$ intensity (radiance) and the luminance contribution as a function of calcination temperature; color chromaticity diagram (b); PL-PLE contour plots of a-YAB powders calcined at $470{ }^{\circ} \mathrm{C}$ (c) and $600{ }^{\circ} \mathrm{C}(\mathrm{d})$. 


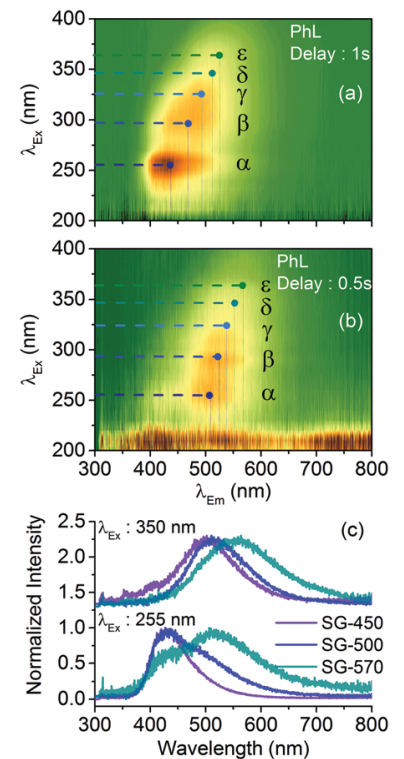

Fig. 6 Time-gated phosphorescence contour plots of SG-500 (a) and SG-570 (b) a-YAB powders. The data in (a) represent the phosphorescence signals at $1 \mathrm{~s}$ after ceasing the excitation, whereas it is at $0.5 \mathrm{~s}$ in (b) as the afterglow intensity is poor for SG-570 due to a higher calcination temperature (ESI, $\dagger$ Fig. S4). The phosphorescence spectral profiles of a-YAB powders under $255 \mathrm{~nm}$ and $350 \mathrm{~nm}$ excitations are presented in (c).

longer wavelength emitting centers with the increase of calcination temperature up to $600{ }^{\circ} \mathrm{C}$ induces the broadening in $\mathrm{PL}$ spectra (Fig. 5d; and ESI, $\dagger$ Fig. S1 and S2).

The relative strengthening of the longer wavelength emitting center could be confirmed from the time-gated phosphorescence plot of higher calcination temperature powders. Fig. 6a shows the phosphorescence contour plot of SG-500, still possessing the dominant contribution from $\alpha$ centers' $\mathrm{S}_{2}$ state, but the relative contribution from $\beta, \gamma, \delta$, $\varepsilon$, etc., also increased notably compared to SG-450 (Fig. 1b). This systematic increase in longer wavelength emitting centers' fraction is more vivid in SG-570 (Fig. 6b). It can also be noticed that the phosphorescence profile is broadened and red shifted in SG-500 and SG-570 powders over SG-450 powder (Fig. $6 \mathrm{c}$ ). This is due to the radiation trapping effect as the longer wavelength emitting centers become more abundant with higher calcination temperature and their absorption overlaps with the emission of shorter wavelength emitting centers as seen from the allowed energy level schemes of the first few prominently deducible emitting centers $(\alpha, \beta, \gamma, \delta$ and $\varepsilon)$

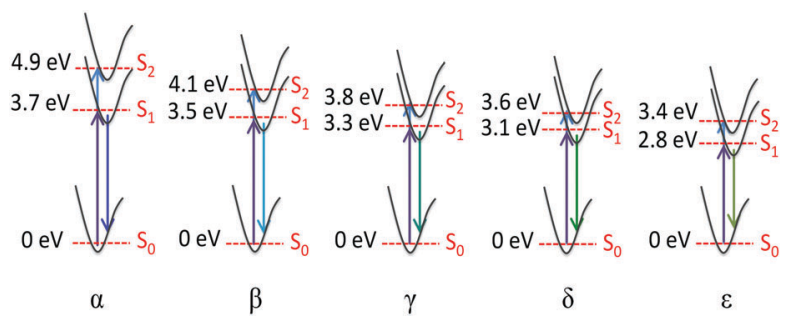

Fig. 7 Allowed electronic transitions and energy levels of the first few prominent emitting centers in a-YAB powders.
Table 1 Color rendering index, $R_{\mathrm{a}}$, correlated color temperature, CCT, luminous efficacy of radiation, LER and luminescence quantum efficiency, QY, of a-YAB powders under $365 \mathrm{~nm}$ LED excitation

\begin{tabular}{lllll}
\hline Samples & CRI $\left(R_{\mathrm{a}}\right)$ & CCT $(\mathrm{K})$ & LER $\left(\operatorname{lm~W}^{-1}\right)$ & iQY $( \pm 5 \%)$ \\
\hline SG-450 & - & $>100000$ & 181 & 32 \\
SG-470 & 82 & 22600 & 215 & 31 \\
SG-500 & 85 & 9900 & 252 & 30 \\
SG-530 & 87 & 6450 & 261 & 34 \\
SG-570 & 86 & 5090 & 275 & 34 \\
SG-600 & 89 & 4370 & 264 & 31 \\
\hline
\end{tabular}

in $\mathrm{a}-\mathrm{YAB}$ powders (Fig. 7). The transition energies were obtained from the PL-PLE measurements of various samples. The relative prominence of longer wavelength emitting centers is also reflected in the absorption profiles of the a-YAB powders, which exhibit a gradual broadening as a function of calcination temperature (ESI, $\dagger$ Fig. S3).

The systematic evolution in PL allows smooth tuning of PL colors from blue to yellow intersecting with white color chromaticity. This is, in particular, an interesting property in view of phosphors for solid-state lighting devices with tunable chromaticity. Moreover, the continuous distribution of PL emissions covering the whole visible range helps to achieve high color rendering ability and reasonable luminous efficacy. ${ }^{24}$ The color rendering index, $R_{\mathrm{a}}$, the correlated color temperature, CCT, the luminous efficacy of radiation (LER) and the luminescence internal quantum efficiency (iQY) values are presented in Table 1 . Both $R_{\mathrm{a}}$ and LER are maximum for the powders calcined at $530-570{ }^{\circ} \mathrm{C}$, which are also optimized powders in view of relative intensity (inset Fig. 5a) and white color chromaticity (CCT $\sim 5500 \mathrm{~K}$; Fig. 5b). The iQY is about $30 \%$ in the powders used, which suggests that a careful tailoring of small PAH molecules trapped in aluminium borate matrices can potentially lead to efficient phosphors.

\section{Conclusions}

In summary, time-gated triplet-state spectroscopy has been used to construct the electronic structures of organic luminophores trapped in the a-YAB glassy matrix (micrometer-sized powders). The results successfully explained the luminescence mechanism in a-YAB powders in more detail. TD-DFT analysis revealed that among directly identified $\mathrm{PAH}$ compounds via magnetic resonance techniques, the phenalene-related compounds exhibit most resemblance with the experimentally derived electronic structure of the luminophores in the a-YAB matrix. The results demonstrated that time-gated spectroscopy is a vital technique to understand the complex PL mechanism in such defect-induced phosphors and to specify the nature of the confined molecular impurities in the rigid network, which play the role of PL emitting centers.

\section{Experimental}

\section{(a) Material synthesis}

The a-YAB powders with chemical composition close to the $\mathrm{YAl}_{3}\left(\mathrm{BO}_{3}\right)_{4}$ compound were obtained by the sol-gel synthesis 
method. In the first solution, $2.7 \mathrm{~mL}$ of $\mathrm{B}\left(\mathrm{OC}_{2} \mathrm{H}_{5}\right)_{3}(99 \%$, Sigma Aldrich) was added in $3.5 \mathrm{~mL}$ of absolute ethanol. The addition of $3.1 \mathrm{~mL} \mathrm{Al}\left(\mathrm{OCH}\left(\mathrm{CH}_{3}\right) \mathrm{CH}_{2} \mathrm{CH}_{3}\right)_{3}(98 \%$, STREM Chemicals) at $50{ }^{\circ} \mathrm{C}$ gives rise to the transesterification reaction among the reactants resulting in the formation of the aluminium borate network. In the second solution, $1.532 \mathrm{~g} \mathrm{Y}\left(\mathrm{NO}_{3}\right)_{3} \cdot 6 \mathrm{H}_{2} \mathrm{O}(99.9 \%$, STREM Chemicals) was dissolved in $0.9 \mathrm{~mL}$ of propionic acid $\left(\mathrm{CH}_{3} \mathrm{CH}_{2} \mathrm{COOH}\right.$, 99\%, Sigma Aldrich) at $50{ }^{\circ} \mathrm{C}$. This solution was then added to the first one, inducing the dissolution of the aluminium borate solid and leading to a translucent yellowish solution. The molar ratio of $\mathrm{Y}: \mathrm{Al}: \mathrm{B}:$ propionic acid is equal to $1: 3: 4: 3$. The resulting solution was then placed under reflux $(2 \mathrm{~h})$ to maximize the hydrolysis and condensation reactions of the reagents. Then, it was evaporated at $70{ }^{\circ} \mathrm{C}$ to obtain a dry resin. This resin was carefully hand-milled before thermal treatments at various calcination temperatures, between 450 and $600{ }^{\circ} \mathrm{C}$, under an $\mathrm{O}_{2}$ atmosphere. The heating rate was kept at $2{ }^{\circ} \mathrm{C} \mathrm{min}^{-1}$ and the soaking duration was one hour.

\section{(b) Experimental methods}

The PL measurement was carried out using a broadband Xe plasma lamp (Energetiq EQ-99X) attached with a Jobin-Yvon UV monochromator for excitation, and the signals were recorded using an Avantes CCD spectrophotometer (AvaSpec-2048 TEC). The spectrometer was calibrated for a relative spectral power distribution using a halogen lamp (Ava-Hal-Cal) and the excitation lamp intensity correction was made using a UV-enhanced Si photo power meter (Thorlabs PM100D). The same setup was used for PL-PLE contour mapping, where the PL was recorded for a series of excitation wavelengths with $5 \mathrm{~nm}$ step resolution. In the case of afterglow contour mapping (PhL-PhLE), the setup including the broadband Xe plasma lamp attached with the monochromator was used for excitation and the signals were recorded using an Acton SpectraPro 2150 Dual Grating Monochromator and a Pixis 100 CCD camera with a manual shutter to cease the excitation. The PhL-PhLE contour represents time-gated phosphorescence signals at $1 \mathrm{~s}(0.5 \mathrm{~s}$ in the case of SG-570) after ceasing the excitation. For the afterglow decay, the same setup was used as above for excitation and emission. The PL thermal stability measurements were carried out using a Sumitomo Cryogenics HC-4E closed cycle He cryostat connected with a Lakeshore 340 Temperature controller. The PL was recorded under continuous excitation of a Xe lamp during controlled heating from $10 \mathrm{~K}$ to $600 \mathrm{~K}$. The UV-visible-NIR reflectance spectra of the powder samples were recorded using a Cary-Eclipse UV-visible-NIR spectrophotometer in diffuse reflectance mode.

\section{(c) TD-DFT analysis}

All DFT calculations have been performed with the ADF (Amsterdam Density Functional) code developed by E. J. Baerends and co-workers ${ }^{25}$ using triple-zeta (TZ2P) basis sets (no frozen core). The S-S electronic transitions, oscillator strengths, and S-T transitions are computed using the $\mathrm{B} \mathrm{LYP}^{26}$ exchangecorrelation potential (20\% Hartree-Fock) and are listed in the ESI $\dagger$ (Table S1).

\section{Conflicts of interest}

There are no conflicts to declare.

\section{Acknowledgements}

This work was carried out under the ANR LuminoPhor-LED project (ANR-14-CE05-0033). The authors would like to thank Prof. Philippe Smet, UGent, for the discussion on phosphor properties.

\section{Notes and references}

1 G. A. Junk and J. J. Richard, Anal. Chem., 1986, 58, 962.

2 S. H. Hong, W. J. Shim and L. Hong, Anal. Methods, 2017, 9, 1361.

3 J. Patarin, Angew. Chem., Int. Ed., 2004, 43, 3878.

4 P. Burner, A. D. Sontakke, M. Salaün, M. Bardet, J.-M. Mouesca, S. Gambarelli, A.-L. Barra, A. Ferrier, B. Viana, A. Ibanez, V. Maurel and I. Gautier-Luneau, Angew. Chem., Int. Ed., 2017, 56, 13995.

5 V. F. Guimarães, L. J. Q. Maia, I. Gautier-Luneau, C. Bouchard, A. C. Hernandez, F. Thomas, A. Ferrier, B. Viana and A. Ibanez, J. Mater. Chem. C, 2015, 3, 5795.

6 A. D. Sontakke, A. Ferrier, P. Burner, V. F. Guimarães, M. Salaün, V. Maurel, I. Gautier-Luneau, A. Ibanez and B. Viana, J. Phys. Chem. Lett., 2017, 8, 4735.

7 I. Villa, A. Vedda, M. Fasoli, R. Lorenzi, N. Kränzlin, F. Rechberger, G. Ilari, D. Primk, B. Hattendorf, F. J. Heiligtag, M. Niederberger and A. Lauria, Chem. Mater., 2016, 28, 3245.

8 (a) L. Vaccaro, A. Morano, V. Radzig and M. Cannas, J. Phys. Chem. C, 2011, 115, 19476; (b) R. B. Soriano, E. Kpatcha, A. M. Jakob, J. W. Merkert, C. M. Carlin and T. A. Schmedake, Appl. Phys. Lett., 2007, 91, 091909; (c) A. M. Jakob and T. A. Schmedake, Chem. Mater., 2006, 18, 3173; (d) T. Hayakawa, A. Hiramitsu and M. Nogami, Appl. Phys. Lett., 2003, 82, 2975.

9 W. H. Green, K. P. Le, J. Grey, T. T. Au and M. J. Sailor, Science, 1997, 276, 1826.

10 (a) T. Ogi, Y. Kaihatsu, F. Iskandar, W.-N. Wang and K. Okuyama, Adv. Mater., 2008, 20, 3235; (b) X. Liu, Y. Qiao, G. Dong, S. Ye, B. Zhu, Y. Zhuang and J. Qiu, J. Electrochem. Soc., 2009, 156, P81.

11 C. K. Lin, Y. Luo, H. You, Z. Quan, J. Zhang, J. Fang and J. Lin, Chem. Mater., 2006, 18, 458.

12 S. K. Lam and D. Lo, Chem. Phys. Lett., 1997, 281, 35.

13 S. Reineke and M. A. Baldo, Sci. Rep., 2014, 4, 3797.

14 F. B. Dias, J. Santos, D. R. Graves, P. Data, R. S. Nobuyasu, M. A. Fox, A. S. Batsanov, T. Palmeira, M. N. Berberan-Santos, M. R. Bryce and A. P. Monkman, Adv. Sci., 2016, 3, 1600080.

15 D. Dzebo, K. Börjesson, V. Gray, K. Moth-Poulsen and B. Albinsson, J. Phys. Chem. C, 2016, 120, 23397.

16 F. B. Dias, T. J. Penfold and A. P. Monkman, Methods Appl. Fluoresc., 2017, 5, 012001. 
17 A. Kohler and H. Bassler, Mater. Sci. Eng., R, 2009, 6, 71.

18 B. El Hamaoui, F. Laquai, S. Baluschev, J. Wu and K. Müllen, Synth. Met., 2006, 56, 1182.

19 A. M. Stoneham, Rev. Mod. Phys., 1969, 41, 82.

20 H. Ohkita, W. Sakai, A. Tsuchida and M. Yamamoto, Macromolecules, 1997, 30, 5376.

21 R. Kabe and C. Adachi, Nature, 2017, 550, 384.

22 H. Sun, Z. Hu, C. Zhong, X. Chen, Z. Sun and L.-L. Bredas, J. Phys. Chem. Lett., 2017, 8, 2393.
23 C. Wang, X.-L. Li, Y. Gao, L. Wang, S. Zhang, L. Zhao, P. Lu, B. Yang, S.-J. Su and Y. Ma, Adv. Opt. Mater., 2017, 5, 1700441.

24 P. F. Smet, A. B. Parmentier and D. Poelman, J. Electrochem. Soc., 2011, 158, R37.

25 G. T. Velde and E. J. Baerends, J. Comput. Phys., 1992, 99, 84. 26 (a) A. D. Becke, J. Chem. Phys., 1993, 98, 1372; (b) C. T. Lee, W. T. Yang and R. G. Parr, Phys. Rev. B: Condens. Matter Mater. Phys., 1988, 37, 785. 